\title{
Comparison of Efficacy of Eye Movement Desensitization and Reprocessing and Cognitive Behavioral Therapy on Depression in Patients with Myocardial Infarction
}

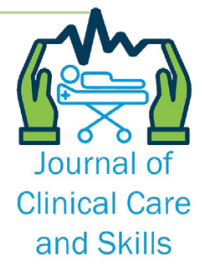

\section{ARTICLE INFO}

\section{Article Type}

Original Research

\section{Authors}

Karimi Z. ${ }^{1} P h D$,

Behnammoghadam M.* PhD, Moazamfard M. ${ }^{2} M S c$, Bashti S. ${ }^{3} M S c$

How to cite this article
Karimi Z, Behnammoghadam M,
Moazamfard M, Bashti S. Compa-
rison of Efficacy of Eye Movement
Desensitization and Reprocessing
and Cognitive Behavioral Therapy
on Depression in Patients with
Myocardial Infarction. Journal of
Clinical Care and Skills. 2020;1(1)
:11-15.

${ }^{*}$ Critical Department, Nursing and Midwifery School, Yasuj University of Medical Sciences, Yasuj, Iran ${ }^{1}$ Operating Room Department, Paramedicine School, Yasuj University of Medical Sciences, Yasuj, Iran ${ }^{2}$ Operating Room Department, Nursing and Midwifery School, Shiraz University of Medical Sciences, Shiraz, Iran

${ }^{3}$ Critical Department, Nursing and Midwifery School, Yasuj University of Medical Sciences, Yasuj, Iran

\section{Correspondence}

Address: Hazrate Zeynab Nursing and Midwifery School, Floor 1, Yasuj University of Medical Sciences, Yasuj, Iran

Phone: +98 (74) 33235041

Fax: +98 (74) 33235041

mbehnam1363@gmail.com

\section{Article History}

Received: December 15, 2017

Accepted: February 20, 2018

ePublished: March 10, 2020

\section{A B S T RAC T}

Aims Depression is a common psychiatric disorder after myocardial infarction. So it is necessary to find the appropriate intervention to reduce depression. This study aimed at comparing the effect of eye movement desensitization and reprocessing (EMDR) and cognitive behavioral therapy (CBT) on depression in patients with myocardial infarction. Materials \& Methods In this clinical trial, 90 eligible patients with myocardial infarction in Imam Sajjad Hospital, Yasuj, Iran were selected by convenience sampling method. Patients were randomly divided to 2 intervention groups and 1 control group by block randomization $(\mathrm{n}=30)$. The EMDR group received the protocol in 8 sessions twice a week for 45 to 90 minutes. The other intervention group received CBT in 10 sessions for 90 minutes twice weekly. The third group did not receive any intervention. Their depression was measured in pre- and post-interventions by Beck Depression questionnaire. The data were analyzed by SPSS 20 software, using descriptive statistics, Fisher's exact test, Chi-square test, paired $t$ test, analysis of variance (ANOVA), and Tukey's post hoc test.

Findings There was a significant difference in the mean difference $(-8.06)$ of depression scores between intervention groups of EMDR and CBT $(\mathrm{p}<0.001)$. EMDR showed a greater reduction in the mean depression values in comparison with the CBT. A significant difference was observed in the mean score depression before and after intervention in both CBT and EMDR groups $(\mathrm{p}<0.001)$.

Conclusion Both EMDR and cognitive behavioral therapy methods reduce depression of patients with myocardial infarction patients, but EMDR is more effective.

Keywords Myocardial Infarction; Eye Movement Desensitization and Reprocessing; Depression; Cognitive-Behavioral Therapy

\section{T A T I O N L I N KS}

[1] Executive summary: Heart disease and stroke statistics--2010 update: A report from the American Heart Association [2] Effects of mental stress on coronary epicardialvasomotion and flow velocity in coronary artery disease: Relationship with hemodynamic stress responses [3] Eye movement desensitization and reprocessing as an adjunctive treatment of unipolar depression: A Controlled Study [4] Efficacy of eye movement desensitization and reprocessing on depression in patients with myocardial infarction in a 12-month follow up [5] Depression and cardiovascular disease: A clinical review [6] Depression following myocardial infarction [7] DMyocardial Infarction and Depression [8] Anxiety treatment by eye movement desensitization and reprocessing in patients with myocardial infarction [9] The impact of cognitive behavioral group training on event -free survival in patients with myocardial infarction the Enrichd experience [10] Effect of eye movement desensitization and reprocessing on depression in patients with myocardial infarction [11] Effect of cognitive-behavioral group therapy on the depression and anxiety in patients with myocardial infarction [12] EMDR and CBT for cancer patients: Comparative study of effects on PTSD, anxiety, and depression [13] A randomized clinical trial of eye movement desensitization and reprocessing (EMDR), fluoxetine, and pill placebo in the treatment of posttraumatic stress disorder: Treatment effects and long-term maintenance [14] Cognitive behaviour therapy versus eye movement desensitization and reprocessing for post-traumatic disorder: Is it all in the homework then? [15] The role of eye movement desensitization and reprocessing (EMDR) therapy in medicine: Addressing the psychological and physical symptoms stemming from adverse life experiences [16] Integrating EMDR into an evolutionarybased therapy for depression: A case study 


\section{Introduction}

Coronary heart disease is a complicated process with atherosclerosis in coronary arteries, including angina pectoralis, myocardial infarction, silent myocardial ischemia, heart failure, and coronary death [1]. Psychological and social factors (anxiety and depression) are involved in the early development of atherosclerosis, acceleration of acute myocardial infarction, and sudden cardiac death [2]. Almost 340 million people worldwide have suffered from depression at least once [3].

Depression is a common psychiatric disorder following myocardial infarction (MI) and is a strong and independent risk factor for mortality after myocardial infarction [4]. Depression is a potent indicator of survival after myocardial infarction and the mortality rate of depressed patients is 3 times greater after myocardial infarction. Clinically, the post-MI prominent depressive symptoms have been observed in $40 \%$ to $65 \%$ of the patients. Depression increases health care costs, decreases fertility, and reduces the quality of life of patients [5]. Preventive measures, as well as treatments, for depression after MI could reduce the risk of mortality, increase the acceptance of treatment and rehabilitation methods, upgrade and maintain the health level and quality of life, prevent high-risk behavior, and prevent the negative consequences of depression in heart disease; thus, prevention and treatment of depression are very important for all cardiac patients [6].

Several therapeutic procedures exist for the treatment of depression after MI, including cardiac rehabilitation, social support, cognitive behavioral therapy, and anti-depressants [7].

One of these cognitive behavioral therapies includes eye movement desensitization and reprocessing (EMDR), a technique in which the therapist asks the client to recall disturbing memories by moving eyes, according to a regular schedule. As a result of decreased arousal, thoughts are once again organized [8]. Another method of treating depression is cognitive-behavioral therapy with confirmed efficacy. In this method, the patient is encouraged to consider the association between negative thoughts and feelings of depression as a hypothesis that should be tested and to use negative behaviors as a benchmark for validity or accuracy of those thoughts [9].

In a study conducted by Hofmann et al., 42 patients with depression were divided to 2 groups. One group received cognitive behavioral therapy and the other group received complementary EMDR in addition to cognitive-behavioral therapy. The results showed that the mean depression significantly reduced in both groups, but patients treated by EMDR had a greater decrease in depression scores [3]. But, the researchers did not find any research to compare the effect of EMDR alone with cognitive-behavioral therapy on depression.

Journal of Clinical Care and Skills
Regarding the high prevalence of depression in patients with myocardial infarction, effective treatment for depression should be taken into consideration by the medical staff. So, the present study aimed at comparing the effect of EMDR with cognitive behavioral therapy (CBT) on depression in patients with myocardial infarction.

\section{Materials and Methods}

The present clinical trial was performed on 90 eligible patients with myocardial infarction (MI) in Imam Sajjad Hospital, Yasuj, Iran. The sample size was calculated based on a similar study, where 30 patients were included in each group, making a total of 90 patients. The inclusion criteria included patients with myocardial infarction, diagnosed by a physician, patients with more MI than once having passed the acute phase with moderate (Rating of 1729) to severe depression (Rating of 30-63), patients with stable hemodynamic status, and patients without the history of mental illness, drug addiction, strabismus, and visual problems. Exclusion criteria included patients unable to tolerate EMDR treatment or patients who did not cooperate with the therapist.

All patients were selected by convenience sampling method. Patients were randomly divided to 2 intervention groups and 1 control group by block randomization. First, the study aims and number of meetings were explained for participants; then, informed consent was obtained from them.

Instruments consisted of the following 2 tools:

1) A questionnaire containing demographic information, such as age, sex, educational level, smoking history, and marital status, collected by the researcher.

2) Beck depression questionnaire; a self-report questionnaire containing 21 questions. The questionnaire is designed based on a Likert scale and includes 21 questions, expressing the symptoms and feelings of depressive patients. The answers are scored on a 4-point scale (0-3) according to the severity of symptoms that result in a total score of 0 to 63 that are classified as following: 0 to 9 as no or minimal depression, 10 to 16 as minor depression, 17 to 29 as moderate depression, and 30 to 63 as severe depression. Beck et al. reported the internal consistency and validity of the scale at $92 \%$ and testretest reliability of 1 week at $75 \%$ and the correlation of variables from $30 \%$ to $76 \%$ [10].

In a study by Khodai et al., the internal consistency coefficient was 0.72 to 0.93 with the mean of 0.86 [11].

The Eye Movement Desensitization and Reprocessing group received the protocol in 8 sessions twice a week for 45 to 90 minutes in aquiet room in the hospital. The other intervention group received cognitive behavioral therapy in 10 sessions for 90 minutes twice weekly by a clinical psychologist in Imam Sajjad Hospital, Yasuj. All 
patients in 3 groups used Oxazepam as routine. The third group did not receive any intervention by the researcher. Their depression was measured in pre- and post-interventions by Beck Depression questionnaire.

The contents of meetings aimed at identifying challenge and change of the negative cognitions of the team members, because the group members experienced the same mental disorders such as anxiety and depression due to myocardial infarction. After obtaining approval from the Ethics Committee of Yasuj University of Medical Sciences (Ethics code ir.yums.rec.1395.26) and registering the protocol of the study in Iran registry of clinical trials (IRCT2016052428017N2) and getting the patient's written informed consent, the study intervention began.

Cognitive behavioral therapy method: In the first stage, patients were consulted about their expectations of treatment and the conditions of participation in meetings, duration, and time of meetings and verbally agreed with them. Also, in this meeting, it was stated that the physiological, cognitive, and behavior processes interact with each other in human beings, and emotions have physiological, cognitive, and behavioral components; then, patients were familiarized with the most provocative thought or belief and its emotional consequences. The participants were assisted to recognize their superficial beliefs during weeks between meetings and record them in a threecolumn structure. The third session included a theoretical discussion about the role of knowledge in 2 negative emotions of anxiety and depression. After they recorded several provocative thoughts or beliefs and the emotional consequences, they were taught to recognize negative schemas or core beliefs, using the vertical arrow method. In order to achieve an overall understanding of the problem and the relationship between false beliefs, the participant were taught about categorizing the beliefs in 1 class, listing the beliefs, and designing a cognitive map. When beliefs were organized, the participants were taught about methods for dealing with beliefs, such as comparing beliefs with objective facts and assessing the usefulness of any belief and empirical test. The goal of this part of the program was to reach the fact that their beliefs were false. After this step, the different methods of changing beliefs were instructed to participants, including reversing or prohibiting the initial interaction, such as creating the opposite belief, self-control approach (punishment, self-reward), and optional cortical inhibition. In addition, in each training session, they were given tasks about the presented issues to practice between sessions [12]. Then, depression test was completed after completion of the training sessions.

Eye movement desensitization and reprocessing method: The EMDR therapy included 8 phases. Each

Journal of Clinical Care and Skills session time took 45 to 90 minutes. In the reprocessing phases, the patient was instructed to recognize a picture, representing the most terrible part of the cardiac incident; a negative unreasonable self-belief related to the picture, a positive adaptive cognition, emotions, and employee body sensations. Then, at first, focusing on the picture, negative belief, and sensation, the patient was guided according to standardized protocols to simultaneously move his eyes back and forward, following the therapist's finger as they moved across their field of vision for a set of about 24 to 36 seconds. After the set, the patient reported any new associations that might have emerged. Such associations, generally, became the focus of the next set of double attention, or were guided by the physician. This procedure continued until the target memory was desensitized. After that, more eye movement sets were used, while the patient was thinking of a recognized adaptive belief. This was repeated until the new statement felt proper to the patient, and until all physical disorder was dissipated. Over the sessions, this treatment method was used to address memories of the cardiac incident, and associated present triggers, as well as anticipatory anxiety related to possible future incidents [13].

According to Kolmogorov-Smirnov test, all variables had a normal distribution.

The data were analyzed by SPSS 20 software, using descriptive statistics, Fisher's exact test, Chi-square test, paired t test, analysis of variance (ANOVA), and Tukey's post hoc test.

\section{Findings}

Patients' age ranged between 33 to 69 years old and the mean age of the patients was 47.62 \pm 8.93 . Totally, $72.22 \%$ of the patients $(n=65)$ were male and $27.77 \%(n=25)$ were female; $91.02 \%(n=82)$ were married and $8.88 \%(n=8)$ were single. In terms of education level, $57.77 \%(n=52)$ had lower than high school education, 29.97\% $(n=27)$ were bachelors, and $12.21 \%(\mathrm{n}=11)$ had a master's degree. Generally, $60.0 \%(n=54)$ of the patients had no history of smoking and only $40 \%(n=36)$ were smoker. No statistically significant difference was observed among 3 groups in terms of demographic characteristics such as gender, education, marital status, and smoking and the groups were homogeneous ( $\mathrm{p}>0.05$; Table 1$)$.

The mean depression before intervention was not significantly different among 3 groups. The mean difference in depression after intervention in 3 groups showed a significant difference $(\mathrm{p}<0.001)$. The differences in mean depression after the intervention between the control group and EMDR group had no statistically significant difference $(p>0.05)$. But, the mean difference in depression (9.73) between CBT and control groups had statistically significant difference $(\mathrm{p}<0.001)$. A

Winter 2020, Volume 1, Issue 1 
significant difference in the mean difference in depression (-8.06) was seen between EMDR and CBT groups $(\mathrm{p}<0.001)$. On the other hand, EMDR showed a greater reduction in the mean depression values in comparison with the CBT $(\mathrm{p}<0.001$; Table 2).

A significant difference was observed in the mean score depression before and after intervention in both CBT and EMDR groups; but, this mean score in the control group did not show significant difference in the post-intervention period compared with the pre-intervention period (Table 2).

Table 1) Demographic characteristics of the studied patients $(\mathrm{N}=30 \text { in each group })^{a}$

\begin{tabular}{|c|c|c|c|c|}
\hline $\begin{array}{c}\text { Variab } \\
\text { les }\end{array}$ & $\begin{array}{l}\text { Control } \\
\text { Group }\end{array}$ & $\begin{array}{l}\text { EMDR } \\
\text { Group }\end{array}$ & $\begin{array}{c}\text { CBT } \\
\text { Group }\end{array}$ & P-value \\
\hline \multicolumn{5}{|l|}{ Sex } \\
\hline Male & 18 (59.9\%) & $21(69.9 \%)$ & $20(66.6 \%)$ & \multirow{2}{*}{0.734} \\
\hline Female & $12(40.1 \%)$ & $9(29.9 \%)$ & $10(33.3 \%)$ & \\
\hline \multicolumn{5}{|c|}{ Educational level } \\
\hline $\begin{array}{l}\text { High } \\
\text { school }\end{array}$ & 15 (49.9\%) & 18 (59.9\%) & $20(66.6 \%)$ & \multirow{3}{*}{0.635} \\
\hline Bachelor & $8(26.6 \%)$ & $10(33.3 \%)$ & $9(29.9 \%)$ & \\
\hline MA & $7(23.3 \%)$ & $2(6.6 \%)$ & $1(3.3 \%)$ & \\
\hline \multicolumn{5}{|c|}{ Marital status } \\
\hline Single & $5(16.6 \%)$ & $2(6.6 \%)$ & $3(9.9 \%)$ & \multirow{2}{*}{0.932} \\
\hline Married & 25 (83.2\%) & $28(93.2 \%)$ & 27 (89.9\%) & \\
\hline \multicolumn{5}{|l|}{ Smoking } \\
\hline Yes & $10(33.3 \%)$ & $13(43.2 \%)$ & $14(46.6 \%)$ & \multirow{2}{*}{0.121} \\
\hline No & $20(66.6 \%)$ & $17(56.6 \%)$ & $16(53.2 \%)$ & \\
\hline
\end{tabular}

Table 2) Mean and standard deviation of depression score before and after intervention in the studied groups

\begin{tabular}{|lccc|}
\hline \multicolumn{1}{c}{ Groups } & $\begin{array}{c}\text { Before } \\
\text { intervention }\end{array}$ & $\begin{array}{c}\text { After } \\
\text { intervention }\end{array}$ & P-value \\
\hline CBT & $34.76 \pm 6.25$ & $23.60 \pm 5.46$ & 0.001 \\
EMDR & $37.86 \pm 6.96$ & $21.63 \pm 7.00$ & 0.001 \\
Control & $35.60 \pm 6.55$ & $30.66 \pm 6.12$ & 0.754 \\
\hline
\end{tabular}

\section{Discussion}

This is the first study that compares the efficacy of eye movement desensitization and reprocessing (EMDR) and cognitive behavioral therapy (CBT) on depression in patients with acute myocardial infarction. One of the important findings of the present research is that both procedures were effective in reducing depression in patients with MI, and in comparison, the effectiveness of EMDR was more than CBT.

Capezzani et al. conducted a comparative study on the impact of Eye Movement Desensitization and Reprocessing (EMDR) and cognitive behavior therapy on anxiety, and depression in patients with cancer. The results showed that both interventions reduce anxiety and depression in the studied patients that are consistent with the results of this study [12]. In a study conducted by Hofmann et al., 42 patients with depression were divided to 2 groups. One group received cognitive behavioral therapy and the other group received complementary EMDR in addition to cognitive-behavioral therapy. The results showed that depression significantly reduced in both groups, but patients treated by EMDR had a greater decrease in depression scores [3]. In a clinical trial by van der Kolk et al., the efficacy of fluoxetine, EMDR, and placebo were examined in patients with post-traumatic stress disorder. The results showed that the group that received EMDR treatment significantly reduced depression scores compared to the group that received fluoxetine [13]. Results of the study conducted by Ho and Lee have shown that therapeutic effects of EMDR on the treatment of depression were significantly stronger than cognitive-behavioral therapy [14].

The results of a review study conducted by Shapiro in 2014 have shown that EMDR had positive effects in the treatment of emotional trauma and other negative experiences of life in 24 clinical trials. Seven of these studies showed that this method results in faster and more effective treatment of negative life experiences and feelings than cognitivebehavioral therapy [16]. The results of this study are consistent and confirmatory with the present study. Commenting on these results, we can say that important stressful events not only occurs in stimulating periods of depression, but such memories can maintain and continue depression; so, processing the abnormal stored memories, such as stressful events with eye movement desensitization and reprocessing in patients with depression may significantly improve symptoms of depression [3].

Depression is a multifactorial disease and stressful life events can cause these conditions.

Shapiro explains the therapeutic effect of EMDR based on the data processing model data processing as follows: the brain is a physiological information processing system, which will generally direct any new information to an appropriate adaptive situation. But, when uncomfortable or traumatic experiences are not sufficiently processed, that thought remains as disturbing.

These disturbing memories create abnormal continuous reactions and brain can process them with eye movement or other bilateral stimulations adaptively [16].

Compared to the CBT method, one of the advantages of this approach is that the number of sessions is less, and its effectiveness is durable. Another advantage of the EMDR treatment method is that it is a new and safe method and does not require homework [8, 4].

Comparison of the effect of EMDR treatment and pharmacotherapy on the level of anxiety and depression is recommended.

It is suggested that another study be carried out considering the mixed group (EMDR, CBT), as well as the effectiveness of these therapies in a 6 month follow-up.

One of the limitations of the present study was that all 3 groups were under medical treatment and the 
removal of medication was not possible.

\section{Conclusion}

Both EMDR and cognitive behavioral therapy methods reduce depression of patients with myocardial infarction patients, but EMDR is more effective.

Acknowledgments: The authors appreciate the research deputy of Yasuj University of Medical Sciences because of their support and also thank the nurses of Heart and Vessel Yasuj Hospital, who sincerely cooperated in all the procedures of this study. Also, the authors appreciate all patients and Francine Shapiro for the assistance provided with the research.

Ethical Permission: The Ethics Committee of Yasuj University of Medical Sciences approved the study with code IR.yums.rec.1395.26.

Conflicts of Interests: The authors declare that there is no conflict of interests.

Authors' Contribution: Karimi Z. (First author), Methodologist/Original researcher/Statistical analyst/Discussion author (30\%); Behnammoghadam M. (Second author), Introduction author/Methodologist/Original

researcher/Statistical analyst/Discussion author (30\%); Moazamfard M. (Third author), Assistant (20\%); Bashti S. (Fourth author), Assistant (20\%)

Funding/Support: The present research was funded by the deputy of research and technology of Yasuj University of Medical Sciences, Iran.

\section{References}

1- Lloyd-Jones D, Adams RJ, Brown TM, Carnethon M, Dai S, De Simone G, et al. Executive summary: Heart disease and stroke statistics--2010 update: A report from the American Heart Association. Circulation. 2010;121(7):948-54.

2- Kop WJ, Krantz DS, Howell RH, Ferguson MA, Papademetriou V, Lu D, et al. Effects of mental stress on coronary epicardialvasomotion and flow velocity in coronary artery disease: Relationship with hemodynamic stress responses. J Am Coll Cardiol. 2001;37(5):1359-66.

3- Hofmann A, Hilgers A, Lehnung M, Liebermann P, Ostacoli L, Schneider W, et al. Eye movement desensitization and reprocessing as an adjunctive treatment of unipolar depression: A Controlled Study. J EMDR Pract Res. 2014;8(3):103-12.

4- Behnam-moghadam M, Behnam-moghadam A, Salehian
T. Efficacy of eye movement desensitization and reprocessing on depression in patients with myocardial infarction in a 12-month follow up. Iran J Crit Care Nurs. 2015;7(4):221-6.

5- Hare DL, Toukhsati SR, Johansson P, Jaarsma T. Depression and cardiovascular disease: A clinical review. Eur Heart J. 2014;35(21):1365-72.

6- Frasure-Smith N, Lesperance F, Talajic M. Depression following myocardial infarction. Impact on 6-month survival. JAMA. 1993;270(15):1819-25.

7- Bagherian-Sararoudi R, Saneei H, Baghbanian A. Myocardial Infarction and Depression. J Isfahan Med Sch. 29(127):102-14. [Persian]

8- Moradi M, Zeighami R, Behnam Moghadam M, Javadi HR, Alipor M. Anxiety treatment by eye movement desensitization and reprocessing in patients with myocardial infarction. Iran Red Crescent Med J. 2016;18(12):1-5.

9- Saab PG, Bang H, Williams RB, Powell LH, Schneiderman $\mathrm{N}$, Thoresen $\mathrm{C}$, et al. The impact of cognitive behavioral group training on event -free survival in patients with myocardial infarction the Enrichd experience. J Psychosom Res. 2009;67(1):45-56.

10- Behnam-moghadam M, Alamdari AK, Behnammoghadam A, Darban F. Effect of eye movement desensitization and reprocessing on depression in patients with myocardial infarction. Glob J Health Sci. 2015;7(6):258-62.

11- Khodai S, Khazai K, Kazemi T, Ali Abadi Z. Effect of cognitive-behavioral group therapy on the depression and anxiety in patients with myocardial infarction. Mod Care J. 2012;9(4):364-70. [Persian]

12- Capezzani L, Ostacoli L, Cavallo M, Carletto S, Fernandez I, Solomon R, et al. EMDR and CBT for cancer patients: Comparative study of effects on PTSD, anxiety, and depression. J EMDR Pract Res. 2013;7(3):134-43.

13- Van Der Kolk BA, Spinazzola J, Blaustein ME, Hopper JW, Hopper EK, Korn DL, et al. A randomized clinical trial of eye movement desensitization and reprocessing (EMDR), fluoxetine, and pill placebo in the treatment of posttraumatic stress disorder: Treatment effects and longterm maintenance. J Clin Psychiatry. 2007;68(1):37-46.

14- Ho MSK, Lee C. Cognitive behaviour therapy versus eye movement desensitization and reprocessing for posttraumatic disorder: Is it all in the homework then?. Eur Rev Appl Psychol. 2012;62(4):253-60.

15- Shapiro F. The role of eye movement desensitization and reprocessing (EMDR) therapy in medicine: Addressing the psychological and physical symptoms stemming from adverse life experiences. Perm J. 2014;18(1):71-7.

16- Valery Krupnik. Integrating EMDR into an evolutionary-based therapy for depression: A case study. Clin Case Rep. 2015;3(5):301-7. 